\title{
Female mating preference for large size in Coelopa frigida (seaweed fly)
}

\author{
A. S. GILBURN, S. P. FOSTER, \& T. H. DAY \\ Department of Genetics, University of Nottingham, Queens Medical Centre, Nottingham NG7 2UH, U.K.
}

\begin{abstract}
Female mating preferences were studied in the seaweed fly, Coelopa frigida. The female acceptance/rejection response to the intial mount by randomly chosen males was scored and male mating success determined by the subsequent production of progeny. The sizes of males and females, as well as their genotypes at the alcohol dehydrogenase $(A d h)$ locus, were determined. Both adult size and the $A d h$ locus are known to be strongly associated with a large chromosomal inversion system. Stepwise multiple regression analyses revealed no association between male mating success and male $A d h$ genotype, but a stong association was found with male size. Female mating preferences appear to exert strong directional selection in favour of large males. There was also an association between female acceptance rate and female $A d h$ genotype, which is likely to be a consequence of inversion karyotype rather than the $A d h$ locus. It is suggested that the discrepancy between the mean size of males in natural populations and the preferred male size indicates that the forces of natural (viability) selection and sexual selection act in opposition.
\end{abstract}

Keywords: Coelopa frigida, female mate choice, sexual selection.

\section{Introduction}

One of the most controversial problems in current biological research is the evolution of male secondary sexual characteristics and of the female mating preferences acting on them, particularly in polygynous species in which males do not provide resources to the female or offspring (see recent reviews by Kirkpatrick \& Ryan, 1991; Maynard-Smith, 1991). Three main schools of thought exist as to how sexual selection by female choice occurs in such species. In the first, the so-called 'good genes' models (Maynard-Smith, 1956; Zahavi, 1975; Andersson, 1982; Hamilton \& Zuk, 1982 ), females are assumed to prefer to mate with males that lead to their offspring having high viability. In consequence females should mate with males that carry genes conferring high viability, or that carry gene complexes complementary to their own; the female mating preference is indirectly adaptive, with natural and sexual selection acting in concert. The second idea derives from Fisher (1930) and was first genetically modelled by O'Donald (1980), Lande (1981) and Kirkpatrick (1982). Genetic variation is assumed to be present at the loci determining the preference and the trait. Non-random mating between the most discriminating females and the most extreme males leads to a genetic correlation between the preference and the trait. Selection acting on the male character will then result in a correlated response in the female mating preference. This process is eventually counter balanced by natural selection. In this case, the female mating preference is maladaptive because it directly opposes natural selection. One subset of the good genes models, the viability indicator models, can also lead to extreme development of the male character as long as natural selection and sexual selection are not stabilizing and both act in the same direction (Zahavi, 1975; Hamilton \& Zuk, 1982; Kirkpatrick, 1986). Even though extreme development of the male character itself may be maladaptive, female mating preferences for the extreme forms of the character can still be adaptive if the male trait is genetically correlated with a trait that increases viability.

A third possible mechanism by which female choice could have evolved is by direct selection acting on the preference itself. By choosing, a female gains an advantage herself, perhaps in terms of increased survival, fecundity or fertility even when there is no transfer of nutrients (see recent reviews by Reynolds \& Gross, 1990; Balmford \& Read, 1991; Kirkpatrick \& Ryan, 1991).

Distinguishing between these alternatives is a chal- 
lenge that faces students of evolution, and the results presented here are the first in a study that attempts to meet this challenge.

Seaweed flies (Coelopa frigida) are polygynous and live in seaweed stranded on beaches around the coastline of the north Atlantic and North Sea. There is no nuptial feeding and K. D. Pitafi has been unable to detect any weight changes in males or females (K. D. Pitafi, unpublished results) following repeated mating; males do not seem to transfer nutrients with their sperm during copulation. The mating success of $C$. frigida males has been found to be influenced by a chromosomal inversion system (Butlin et al., 1982b; Day et al., 1987; Foster, 1989). If male mating success is a direct result of female preferences, why are females choosing their mates on the basis of the male's inversion karyotype? Females may be exercising mate choice on the basis of an adaptive male character associated with the inversion system. Alternatively, females may be preferring to mate with males carrying a maladaptive character, either because it acts as a viability indicator or because the preference gene is genetically correlated with the preferred trait. If the male trait is maladaptive then a sexual dimorphism should evolve. Evidence that the female mating preference may be adaptive in seaweed flies has been found by Crocker \& Day (1987). Females allowed a choice of mate produced offspring with higher egg-to-adult survival than females given no choice. There is as yet no evidence that the preference itself is adaptive in Coelopa.

In order to distinguish between the various mechanisms by which female choice may have evolved, it is important to determine what is the subject of their choice. What characters associated with the inversion system are potential candidates to be the preferred male trait? If the female mating preference has evolved either through a genetic correlation with the male trait or by a viability indicator mechanism, there should be a sexually dimorphic character determined by the inversion that is maladaptive in males. One such character is adult size. The phenotypic variation in adult size is very much greater in males than females (Butlin et al., 1982a,b; Day et al., 1982), and it is the largest males that consistently exhibit the lowest survival from egg to adult (Collins, 1978; Butlin et al., 1982a, 1984; Butlin \& Day, 1989). A very much weaker candidate to be the preferred character is the genotype at the Adh locus, which was used by Engelhard et al. (1989) and Foster (1989) to study male mating success. While they obtained evidence for disassortative mating with respect to this locus, they interpreted their results in terms of the chromosome I inversion system with which Adh is strongly associated (Day et al. 1982). As the chromosome I inversion system is very large, com- prising approximately 10 per cent of the entire genome (Aziz, 1975; Day et al., 1982), it is difficult to distinguish between the effects of size, the $A d h$ locus and the inversion system. There is known to be strong linkage disequilibrium associated with this inversion system (Collins, 1978; Day et al., 1982, 1983), no recombination in heterokaryotypes or in males (Day et al., 1982), and strong heterokaryotypic advantage (Butlin et al., 1982b). Under these circumstances there would seem to be the ideal situation for the operation of a viability indicator system.

The aim of the present study was to determine whether either male size or Adh genotype is the character associated with the chromosome I inversion system that is primarily assessed by females. This was carried out under two distinct mating conditions. In the first, pairs of adults were allowed $5 \mathrm{~h}$ in which to mate. Previous results have shown that this is ample time for many mating attempts to be made. In the second set of experiments only the first mount by the male in each pair was considered, and male rejection was intentionally excluded.

\section{Materials and methods}

\section{Experimental design}

The flies used in the single mount experiment were collected as larvae from St Mary's Island on the northeast coast of England during December 1989 and January 1990 . Virgin flies of both sexes were collected and stored at $4^{\circ} \mathrm{C}$ until required.

Males were transferred individually to freshly minced seaweed for 2 days sex-deprivation at $26^{\circ} \mathrm{C}$. It was found that males were more active sexually if they were separated during this period. The females were also transferred to $26^{\circ} \mathrm{C}$ for 2 days, but were kept in groups of 50 on 0.5 per cent mannitol solution. Flies were then placed in pairs of one male and one female in a mating chamber at approximately $22^{\circ} \mathrm{C}$ (see Day et al., 1990). When the male mounted the female her reaction was noted and the flies separated immediately the male dismounted. The mating behaviour of seaweed flies, and in particular the female acceptance/ rejection response, has been described in detail by Day et al. (1990). If the male failed to mount the female within $5 \mathrm{~min}$, or the male mounted and dismounted without attempting to copulate, then the trial was disregarded. The female acceptance rate was calculated as the proportion of females that were observed to accept the male following his single mount. Manipulations of adults were all carried out without the use of anaesthesia. At the end of the trials the females were placed individually onto freshly minced seaweed for 2 days, 
and allowed to oviposit. The males were stored at $-25^{\circ} \mathrm{C}$ and subsequently their size measured and their Adh genotype determined using starch gel electrophoresis (Butlin et al., 1982b). Sizes of adults were determined by measuring wing length, which is strongly correlated with other body dimensions (see Butlin et al., 1982b). After the 2-day egg-laying period the females were treated in the same manner as the males. Individual pots in which the females had laid eggs were checked for the presence of larvae after a further 2 days. Mating success was calculated as the proportion of adult pairs that mated productively to produce larvae. In contrast to acceptance rate, which refers to one aspect of female behaviour, mating success refers to the complete process of mating.

These single-mount trails measured the response of the female to the very first mount by the male, and male dismounting (male mate choice) was intentionally excluded. A further set of trials was conducted in which pairs of virgin adults were allowed $5 \mathrm{~h}$ in which to mate. The animals were not observed during this time, but other experiments indicated that multiple mounts take place and that the males can and do exercise mate choice. Such extended pair mating experiments yielded information not on female acceptance but on mating success as judged by the subsequent production of progeny larvae. Further experimental details are given in Engelhard et al. (1989).

\section{The generation of selection surfaces and selection differentials}

Stepwise multiple regression analyses were performed using the Generalised Linear Interactive Modelling system (GLIM, Numerical Algorithm Group), and selection differentials estimated from the partial regression coefficients generated. A binomial error distribution has been used throughout the analyses.

Selection surfaces are functions describing the relative fitnesses associated with a continuous distribution of phenotypic values of a quantitative character (Simpson, 1953; Lande, 1979). They can be generated by both parametric and non-parametric analyses, but Lande \& Arnold (1983) suggested that quadratic regression analysis should be used to determine selection surfaces. It has been pointed out, however, by Schluter (1988), that this method can give misleading results, such as falsely suggesting the presence of optima or troughs. Consequently, quadratic regression analysis was used initially to produce selection surfaces which were then compared to selection surfaces produced using GLMS (Schluter, 1988), a system that calculates fitness functions by non-parametric regression using the cubic spline. A cublic spline is a function comprising $n+1$ cubic polynomials joined seamlessly at points corresponding to the $n$ phenotypic values. The value of $\lambda$ controls the smoothing of the spline and is commonly referred to as the 'smoothing parameter'. As $\lambda$ increases the spline becomes smoother. Generalized cross-validation can be used to calculate the value of $\lambda$ with maximum predictive power in each dataset and in doing so removes individual points from the dataset before recalculating the spline. Generalized cross-validation uses short-cut methods to approximate the sum of the predictive errors to produce a generalized cross-validation (GCV) score (Craven \& Wahba, 1979). The value of $\lambda$ that minimizes the GCV score also approximately minimizes the sum of squared deviations between the original spline and the spline calculated with individual data points missing. In the particular analyses carried out, the GLMS program did not produce a minimum GCV score. One likely reason for this was the relatively low number of unique values of the $\mathrm{X}$ variable (male size) compared to the sample size (D. Schluter, personal communication). This problem was avoided by making small random perturbations from all the $X$-values, so breaking the ties. A maximum perturbation of 0.05 was used thereby enabling GLMS to calculate minimum GCV scores. This resulted in a change in wing length of never more than 0.3 per cent.

\section{Results}

In the single mount experiments (but not the extended mating trials) both acceptance rates and mating successes were measured. In each trial the female response was recorded, and whether or not she subsequently produced larvae. As no larvae were produced in trials in which the female rejected the male, we can be confident that the observation of rejection was a reliable indicator of the failure to inseminate. However, it is not true that female acceptance always led to the production of progeny, and in the following sections the differences between acceptance rates and mating success reflect this fact.

\section{Male mating success}

In order to determine whether male mating success (as defined by the production of progeny) covaried with either male size or $A d h$, two multiple regression analyses were performed. In the first, mating success was regressed on male size, then on male size squared, and finally on male $A d h$ genotype. In the second, mating success was regressed on male $A d h$ genotype, then male size, and finally male size squared. These 
Table 1 Stepwise multiple regression analysis of mating success on male $A d h$ genotype and male size. For each multiple regression the male characters were fitted in the order shown. The degrees of freedom (d.f.) and probability $(P)$ associated with each $F$ statistic are given

\begin{tabular}{clrlc}
\hline Experiment & Male character & \multicolumn{1}{l}{$F$} & d.f. & $P$ \\
\hline \multirow{2}{*}{ Single mount } & Size & 3.71 & 1,514 & 0.055 \\
& Size squared & 4.71 & 1,513 & 0.030 \\
& Adh & 1.61 & 8,505 & 0.12 \\
Single mount & Adh & 0.93 & 8,507 & 0.49 \\
& Size & 8.51 & 1,506 & 0.004 \\
& Size squared & 5.22 & 1,505 & 0.023 \\
Five hour & Size & 23.81 & 1,804 & $\ll 0.001$ \\
mating & Size squared & 8.88 & 1,803 & 0.003 \\
& Adh & 1.76 & 8,795 & 0.080 \\
Five hour & Adh & 0.89 & 8,797 & 0.52 \\
mating & Size & 35.05 & 1,796 & $\ll 0.001$ \\
& Size squared & 4.63 & 1,795 & 0.032 \\
\hline
\end{tabular}

results (Table 1) provide no evidence that male mating success covaries with male Adh genotype in either the single mount or the 5-h experiment. However, in both datasets there was a significant association with male size, which may well have a quadratic as well as a linear component. Previous results have revealed an association between $A d h$ genotype and size (Butlin et al., $1982 \mathrm{~b}$ ). What then is the significance of mating success

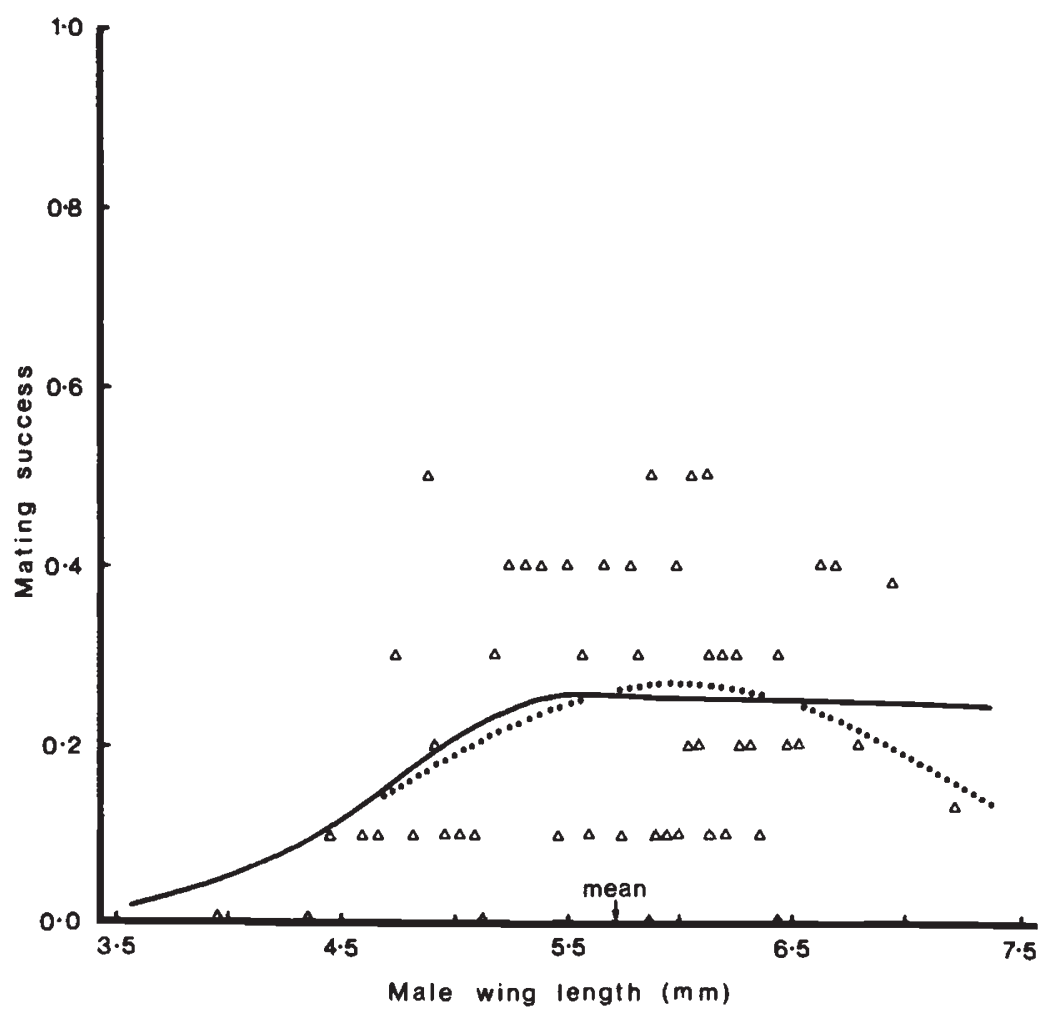

covarying with size but not $A d h$ ? The probable answer is that there is sufficient environmental variance in size within each $A d h$ genotype for mating success to be effectively independent of genotype.

The data were then examined for any association between mating success and the inversion karyotype of the male. The $A d h-B$ allele is always associated with the $\alpha$ form of the inversion on chromosome I, and the $A d h-D$ allele always with the $\beta$-form, but the $A d h-C$ allele does not show such linkage disequilibrium. In consequence the inversion karyotype can only be inferred unambiguously in individuals carrying the $A d h-B$ and $D$ alleles. Using only these animals in the single mount experiment, mating success was regressed on male size and on inversion karyotype. There was a significant association with male size when the variation due to karyotype had been removed $(F=4.97$, d.f. $=1,390, P=0.026)$, but not with karyotype when variation due to male size had been removed $(F=2.09$, d.f. $=2,390, P=0.13$ ). Mating success appears to covary more strongly with size than with karyotype.

Selection surfaces were calculated by both quadratic and non-parametric regression of mating success on male size (see Figs 1 and 2). Although analyses by parametric regression indicated the presence of an optimum, the cubic spline analysis suggested that above a certain male size, mating success reaches a plateau. The only notable difference between the single
Fig. 1 Selection surfaces derived from the single mount experiment. ( $\cdots$..) Selection surface generated by quadratic regression analysis. (- ${ }_{-}$Selection surface generated by non-parametric regression (cubic spline). Surfaces were calculated using the data from individual trials each of which was either 'successful' or 'unsuccessful'. For the purposes of illustration, the means of groups of 10 trials are given. The two groups with the largest males include nine trials. 


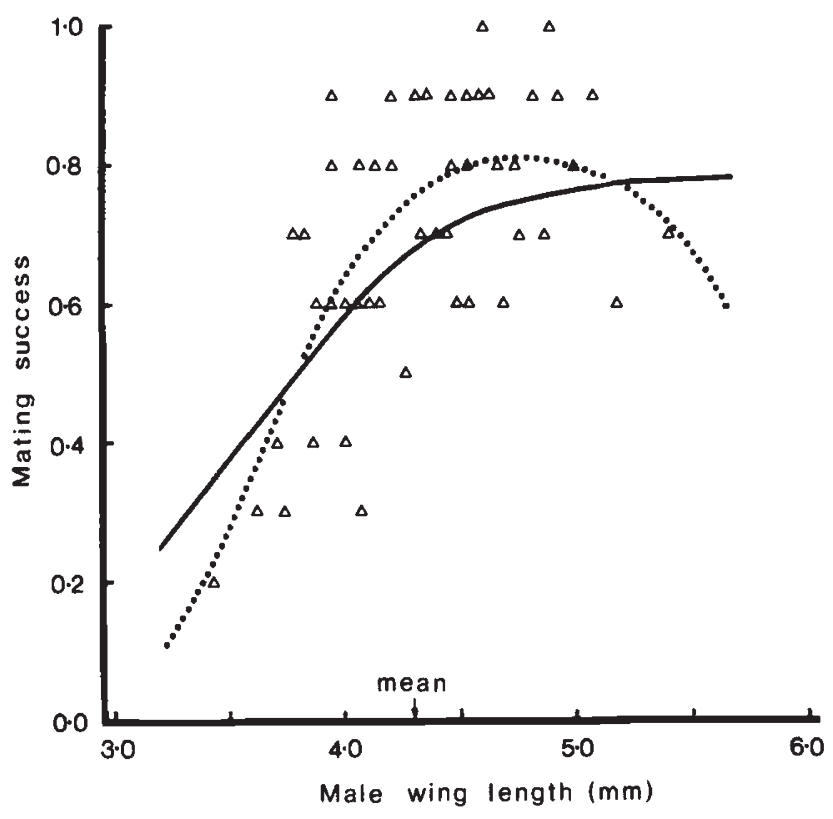

Fig. 2 Selection surfaces derived from the 5-h mating experiment. (…) Selection surface generated by quadratic regression analysis. ( $\longrightarrow$ ) Selection surface generated by non-parametric regression (cubic spline). Surfaces were calculated using the data from individual trials each of which was either 'successful' or 'unsuccessful'. For the purposes of illustration, the means of groups of 10 trials are given.

mount and the 5-h experiments was in the overall level of mating success. Not surprisingly the longer period available for mating in the 5-h experiment resulted in a larger proportion of the females being fertile.

\section{Female mating success}

Stepwise multiple regressions were performed to determine whether female mating success covaries with female size, female $A d h$ genotype and female inversion karyotype. In all cases variation due to male size and male size squared was removed before the female character was fitted. Consider first the single mount experiment (Table 2). There was clearly no association between female mating success and female size but a strong association was revealed between mating success and $A d h$ genotype. When only those females were included whose inversion karyotype could be inferred unambiguously $(A d h B B, B D$ and $D D)$, the association with inversion karyotype was even stronger than with the $A d h$ genotype. It seems then that the chromosome $\mathrm{I}$ inversion system is a major genetic determinant of female mating success. A very different result was obtained from the 5-h mating, in which mating success was strongly associated with female size, but not with the Adh genotype or karyotype. In
Table 2 Stepwise multiple regression analysis of female mating success on female size, female $A d h$ genotype and female chromosome I inversion karyotype. In all cases female mating success was initially regressed on male size and male size squared

\begin{tabular}{clrrl}
\hline Experiment & Female character & $F$ & \multicolumn{1}{l}{ d.f. } & $P$ \\
\hline Single mount & Size & 0.85 & 1,512 & 0.36 \\
& Adh & 2.43 & 10,503 & 0.008 \\
& Karyotype & 6.22 & 2,390 & 0.002 \\
Five hour & Size & 30.17 & 1,795 & $\ll 0.001$ \\
mating & Adh & 1.05 & 7,785 & 0.39 \\
& Karyotype & 1.01 & 2,639 & 0.36 \\
\hline
\end{tabular}

Table 3 Mating successes and acceptance rates of females with different $A d h$ genotypes. Mating success is expressed as the proportion of females of a given genotype that produced progeny. The acceptance rate is the proportion of females that were observed to accept the male following his single mount

\begin{tabular}{llrll}
\hline Experiment & Adh $\begin{array}{l}\text { Number of } \\
\text { females }\end{array}$ & $\begin{array}{l}\text { Mating } \\
\text { success }\end{array}$ & $\begin{array}{l}\text { Acceptance } \\
\text { rate }\end{array}$ \\
\hline Single mount & $B B$ & 86 & 0.34 & 0.81 \\
& $B C$ & 47 & 0.30 & 0.70 \\
& $B D$ & 217 & 0.18 & 0.77 \\
& $C D$ & 53 & 0.36 & 0.70 \\
Five hour & $D D$ & 92 & 0.13 & 0.61 \\
mating & $B B$ & 97 & 0.70 & \\
& $B C$ & 80 & 0.69 & Pairs not \\
& $B D$ & 392 & 0.64 & observed \\
& $C D$ & 74 & 0.66 & \\
\hline
\end{tabular}

the single mount experiment, male mate choice in the form of male dismounting, was intentionally excluded. As Pitafi et al. (1990, unpublished observations) have shown that males prefer to mate with large females, it would appear that male choice can be the major determinant of mating success, so presumably masking the effects of the female's karyotype. The mating successes observed for each female genotype are shown in Table 3. Several points are worth noting. The much higher mating success observed in the 5-h experiment may account for the different $A d h$ genotypes being much more homogeneous, although the general pattern is similar. Secondly, $A d h-B B$ females exhibited a significantly higher mating success than $D D$ homozygotes (using a $2 \times 2$ heterogeneity $\chi^{2}$ on the original numbers of mated and unmated females:

$\chi_{1}^{2}=10.7, P=0.001$ ). 
The rates derived from the much longer mating showed the same trend but are not significant. It would seem that there are genetic determinants associated with the $\alpha$ inversion that result in relatively high mating success. The $\alpha \beta$ heterokaryotypes $(A d h-B D)$ were intermediate although not significantly different from the $\beta \beta$ homozygotes (heterogeneity $\chi^{2}$ between mated and unmated females: $\left.\chi_{1}^{2}=1.14, P=0.29\right)$. Lastly the $C D$ females exhibited an unexpectedly high mating success. As the $\mathrm{C}$ alleles are known to be heterogeneous with respect to the inversion, if mating success is determined solely by karyotypes we should expect $B C$ females to be intermediate between $B B$ and $B D \mathrm{~s}$, and $C D$ s to be intermediate between $B D$ and $D D$ s. In fact the former is observed but the latter is not. $C D$ s showed significantly greater mating success than either $B D \mathrm{~s}\left(\chi_{1}^{2}=8.1, P=0.005\right)$ or $D D$ s $\left(\chi_{1}^{2}=10.4\right.$, $P=0.001)$. There would appear to be a genotypespecific as well as an inversion-specific effect on mating success. This result is consistent with those of Engelhard et al.(1989).

\section{Female acceptance rates}

In the single-mount experiments females were actually observed. Estimates of the proportion of initial mounts that resulted in female acceptance can therefore be obtained for each female genotype (see Table 3). Not surprisingly the acceptance rates were always higher than the mating successes; female acceptance or rejection occurs near the beginning of the mating process (Day et al., 1990), whereas mating success was based on the final outcome of mating - the production of progeny. There is a strong suggestion that the mating success for $B D$ and $D D$ females is somewhat lower than would be predicted from their rates of acceptance (using a $2 \times 5$ heterogeneity table with the numbers of females that accepted and the numbers that were fertile, for each of the five genotypes:

$\chi_{4}^{2}=9.9, P=0.043$ ).

More significantly, there are substantial differences in acceptance rates between genotypes (using the numbers of accepting and rejecting females for each genotype: $\chi_{4}^{2}=12.2, P=0.016$ ), with the acceptance rate by $D D$ females being particularly low. This result concurs with those of Engelhard et al. (1989). A similar association between female acceptance rate and inversion karyotype has been found in Drosophila persimilis (Spiess \& Langer, 1964).

Female acceptance rate was regressed on male size and inversion karyotype, after the variation due to the other had been removed. Female acceptance rate covaried with male size $(F=8.31$, d.f. $=1,390$,
Table 4 Stepwise multiple regression analysis of female mating success (and female acceptance rate in the single mount experiment) on the interaction terms between female size and male size, female $A d h$ genotype and male size, and female chromosome I inversion karyotype and male size

\begin{tabular}{lllrl}
\hline Experiment & $\begin{array}{l}\text { Female } \\
\text { character }\end{array}$ & \multicolumn{1}{l}{$F$} & \multicolumn{1}{c}{ d.f. } & $P$ \\
\hline Single mount & Size & 0.49 & 2,510 & 0.61 \\
(acceptance rate) & Adh & 1.05 & 16,495 & 0.40 \\
& Karyotype & 1.65 & 4,390 & 0.16 \\
Single mount & Size & 1.16 & 2,510 & 0.31 \\
(mating success) & Adh & 0.75 & 16,495 & 0.74 \\
& Karyotype & 0.41 & 4,390 & 0.80 \\
Five hour mating & Size & 2.76 & 2,789 & 0.064 \\
(mating success) & Adh & 0.91 & 12,773 & 0.54 \\
& Karyotype & 0.77 & 4,635 & 0.54 \\
\hline
\end{tabular}

$P=0.004)$ but not with male inversion karyotype $(F=0.53$, d.f. $=2,390, P=0.59)$.

\section{Female choice}

The data were further analysed to discover whether the pattern of female choice, i.e. the association between acceptance rates and male size, covaries with female characters such as size, $A d h$ genotype or inversion karyotype. While female choice is most obviously indicated by the female acceptance/rejection response, we have also carried out the analysis using female mating success. This clearly includes components of the mating process in addition to the observable female response.

In a multiple regression analysis, female acceptance rate (or female mating success) was fitted to male size and male size squared first, then fitted to the female character, and finally fitted to two interaction terms. The first interaction term used was the product of male size and the female character, and the second was the product of male size squared and the female character. There is no evidence that female choice covaries with female $A d h$ genotype or with inversion karyotype in either set of experiments (Table 4). However, there is a suggestion that the size of a female has some effect on her choice in the 5-h experiment, a result that might be a consequence of male choice for large females.

\section{Discussion}

It has been demonstrated that the male character preferred by females exercising mate choice is large size, or of course, some other character correlated with wing length. Among insects, this is not at all exceptional. 
When mate choice is a resource-based system, large males sometimes gain a mating advantage, as for example, in Katydid grasshoppers (Gwynne, 1982) in which males transfer nutrients to the female in the form of a spermatophore. In such species it seems safe to infer that female choice has evolved in response to the increased supply of nutrients available to the female when mating with large males. However, even in nonresource based systems females often prefer to mate with large males. In many such cases female choice appears to be reinforcing the effects of male-male competition (McCawley \& Wade, 1978; Borgia, 1981; Johnson, 1982; Burkhardt \& de la Motte, 1988).

The situation that is most difficult to understand involves a preference for male characters that are maladaptive. In some respects large size appears to be the 'peacock's tail' of seaweed flies. Large males take longer to develop as larvae and so experience a greater risk of being washed out to sea. They are also living in a habitat (decomposing seaweed) previously used by the faster developing larvae (Butlin \& Day, 1984). Estimates of the egg-to-adult survival of $\alpha \alpha$ homokaryotypic males (the largest) have consistently revealed them to be the poorest competitors of all inversion karyotypes (Butlin \& Day, 1984). There seems little doubt that in terms of viability large male size is maladaptive.

However, large size is also associated with increased longevity, there being a roughly twofold difference between $\alpha \alpha$ and $\beta \beta$ males (Butlin \& Day, 1985). Whether this difference results in greater fecundity or fertility for large males independently of the consequences of female choice, is not yet clear.

The combined effects of these diverse forces of natural selection (i.e. excluding sexual selection) is that males of intermediate size are the fittest (see for example Day et al., 1983). In contrast, sexual selection in the form of both female mate choice and male mate choice (Pitafi, 1991; unpublished observations) must operate directionally favouring large size. We therefore have, in seaweed flies, the situation predicted by Fisher (1930) in which natural and sexual selection act in opposition. A similar counteraction of selective forces has been demonstrated in the cricket, Gryllus integer (Cade, 1975), the Túngara frog, Physalaemus pustulosus (Ryan, 1985) and a Trinidad guppy, Poecilia reticulata (Breden \& Stoner, 1987). In these, and doubtless many other species, the actual phenotypic nature of the preferred character presumably approximates to an equilibrium value that is displaced from the optimum as generated by viability selection alone. In the $C$. frigida population studied here, the mean size of males newly collected from the wild was $5.7 \mathrm{~mm}$ whereas female preference was greatest for males in excess of
$5.5 \mathrm{~mm}$ (see Fig. 1). Another indication of this discrepancy is that the mean size of rejected males was 5.54 $\mathrm{mm}$ (S.E. $=0.07$ ) but of those accepted, $5.78 \mathrm{~mm}$ $(\mathrm{S} . \mathrm{E} .=0.03)$ a difference that is highly significant $\left(t_{192}=3.15, P=0.0023\right)$. Data from other populations (to be reported elsewhere) indicate that this difference is consistently observed, and is likely to be a consequence of mate choice.

Having identified the preferred character, are we any nearer understanding the evolution of the preference? At the moment the answer must be no, but there are certain consequences of the preferred character being size that make an answer potentially accessible. A prediction of the Fisherian models is that a genetic correlation should develop between the preference and the preferred character. In most species this prediction has been particularly difficult to test because it presupposes a knowledge of the genetic basis of both variables, and by their very nature they are sex-limited. In Coelopa the $\alpha \beta$ inversion system is known to be a major determinant of male size, and we have now shown that it also influences female acceptance rates. As the $A d h$ locus can be used as a marker of the inversion in both sexes, it should be possible to test for a genetic correlation. This work is in progress.

\section{Acknowledgements}

This work was supported by a grant (GR3/7189) from the Natural Environment Research Council and a studentship (to A. S. Gilburn) from the Science and Engineering Research Council. The helpful advice of Dr M. Beaumont with the statistical analysis was much appreciated.

\section{References}

ANDERSSON, M. B. 1982. Sexual selection, natural selection and quality advertisement. Biol. J. Linn. Soc., 17, 375-393.

AZIZ, J. B. 1975. Investigations into chromosomes 1, 2 and 3 of Coelopa frigida. PhD Thesis. University of Newcastleupon-Tyne.

BALMFORD, A. AND READ, A. F. 1991. Testing alternative models of sexual selection through female choice. Trends Ecol. Evol., 6, 274-276.

BORGIA, G. 1981. Mate selection in the fly Scatophaga stercoraria: female choice in a male-controlled system. Anim. Behav., 29, 71-80.

BREDEN, F. AND STONER, G. 1987. Male predation risk determines female preference in the Trinidad guppy. Nature, 329, 831-833.

BURKHARDT, D. AND DE LA MOTTE, I. 1988. Big 'antlers' are favoured: female choice in stalk-eyed flies. Field collected harems and laboratory experiments. J. Comp. Physiol. A., 162, 649-652. 
BUTLIN, R. K., COLLINS, P. M. AND DAY, T. H. 1984. The effect of larval density on an inversion polymorphism in the seaweed fly Coelopa frigida. Heredity, 52, 415-423.

BUTLIN, R. K., COLLINS, P. M., SKEVINGTON, S. J. AND DAY, T. H. 1982a. Genetic variation at the alcohol dehydrogenase locus in natural populations of the seaweed fly, Coelopa frigida. Heredity, 48, 45-55.

BUTLIN, R. K. AND DAY, T. H. 1984. The effect of larval competition on development time and adult size in the seaweed fly, Coelopa frigida. Oecologia, 63, 122-127.

BUTLIN, R. K. AND DAY, T. H. 1985. Adult size, longevity and fecundity in the seaweed fly, Coelopa frigida. Heredity, 54, 107-110.

BUTLIN, R. K. AND DAY, T. H. 1989. Environmental correlates of inversion frequencies in natural populations of seaweed flies (Coelopa). Heredity, 62, 223-232.

BUTLIN, R. K., READ, 1. L. AND DAY, T. H. 1982b. The effects of a chromosomal inversion on adult size and male mating success in the seaweed fly, Coelopa frigida. Heredity, 49, $51-62$.

CADE, w. 1975. Acoustically orienting parasitoids: fly phonotaxis to cricket song. Science, 190, 1312-1313.

Collins, P. M. 1978. Studies on genetic polymorphism in Coelopa frigida. PhD. Thesis University of Nottingham.

CRAVEN, P. AND WAHBA, G. 1979. Smoothing noisy data with spline functions. Estimating the correct degree of smoothing by the method of generalised cross-validation. Numer. Math., 31, 377-403.

CROCKER, G. AND DAY, T. H. 1987. An advantage to mate choice in the seaweed fly, Coelopa frigida. Behav. Ecol. Sociobiol., 20, 295-301.

DAY, T. H., DAWE, C., DOBSON, T. AND HILLIER, P. C. 1983. A chromosomal inversion polymorphism in Scandinavian populations of the seaweed fly, Coelopa frigida. Hereditas, 99, 135-145.

DAY, T. H., DOBSON, T., HILLIER, P. C., PARKIN, D. T. AND CLARKE, B 1982. Associations of enzymic and chromosomal polymorphisms in the seaweed fly, Coelopa frigida. Heredity, 48, 35-44.

DAY, T. H., FOSTER, S. P. AND ENGElhard, G. 1990. Mating behaviour in seaweed flies (Coelopa frigida). J. Insect Behav., 3, 105-120.

DAY, T. H., MILES, S., PILKINGTON, M. D. AND BUTLIN, R. K. 1987. Differential mating success in populations of seaweed flies (Coelopa frigida). Heredity, 58, 203-212.

ENGELHARD, G., FOSTER, S. P. AND DAY, T. H. 1989. Genetic differences in mating success and female choice in seaweed flies (Coelopa frigida). Heredity, 62, 123-131.

FiSHER, R. A. 1930. The Genetical Theory of Natural Selection. Clarendon Press, Oxford.

FOSTER, S. P. 1989. Selection concerning reproduction in the seaweed fly, Coelopa frigida. PhD Thesis, University of Nottingham.
GWYNNE, D. T. 1982. Mate selection by female katydids (Orthoptera:tettigoniidae) Conocephalus nigropleurum. Anim. Behav., 30, 734-738.

HAMILTON, W. D. AND ZUK, M. 1982. Heritable true fitnesses and bright birds: a role for parasites. Science, 218, 384-387.

JOHNSON, L. K. 1982. Sexual selection in a brentid weevil. Evolution, 36, 251-262.

KIRKPATRICK, M. 1982. Sexual selection and the evolution of female choice. Evolution, 36, 1-12.

KIRKPATRICK. M. 1986. Sexual selection and cycling parasites: a simulation study of Hamilton's hypothesis. J. Theor. Biol., 119, 263-271.

KIRKPATRICK, M. AND RYAN, M. J. 1991. The evolution of mating preferences and the paradox of the lek. Nature, $\mathbf{3 5 0}$, 33-38.

LANDE, R. 1979. Quantitative genetic analysis of multivariate evolution, applied to brain : body size allometry. Evolution, 33, 402-416.

LANDE, R. 1981. Models of speciation by sexual selection on polygenic traits. Proc. Natl. Acad. Sci., U.S.A., 78, 37213725 .

LANDE, R. AND ARNOLD, S. J. 1983. The measurement of selection on correlated characters. Evolution, 37, 1210-1226.

MAYNARD SMITH, J. 1956. Fertility, mating behaviour and sexual selection in Drosophila subobscura. J. Genet., 54, 261-279.

MAYNARD SMITH, J. 1991. Theories of sexual selection. Trends Ecol. Evol., 6, 146-151.

MCCAWLEY, D. E. AND WADE. M. J. 1978. Female choice and the mating structure of a natural population of the soldier beetle Chauliognathus pennsylvanicus. Evolution, 32 , 771-775.

o'donald, P. 1980. Genetic Models of Sexual Selection Cambridge University Press, Cambridge.

PITAFI, K. D. 1991. Male mate choice in seaweed flies, Coelopa frigida. PhD Thesis, University of Nottingham.

PITAFI, K. D., SIMPSON, R., STEPHEN, J. J. AND DAY, T. H. 1990. Adult size and mate choice in seaweed flies. Heredity, 65 , 191-197.

REYNOLDS, J. D. AND GROSS, M. R. 1990. Costs and benefits of female mate choice: is there a lek paradox? Am. Nat., 136, 230-243.

RYAN, M. J. 1985. The Túngara Frog. University of Chicago Press, Chicago.

SCHLUTER, D. 1988. Estimating the form of natural selection on a quantitative trait. Evolution, 42, 849-861.

SIMPson, G. G. 1953. The Major Features of Evolution. Columbia University Press, Columbia.

SPIESS, E. B. AND LANGER, B. 1964. Mating speed and control by gene arrangements in Drosophila persimilis. Evolution, 18, 430-444

ZAHAVl, A. 1975. Mate selection - a selection for a handicap. J. Theor. Biol., 53, 205-214. 